Review

\title{
Circular RNAs: Novel Promising Biomarkers in Ocular Diseases
}

\author{
Nan Guo ${ }^{1^{*}}$, Xiu-fen Liu ${ }^{1 *}$, Om Prakash Pant ${ }^{1 *}$, Dan-Dan Zhou ${ }^{2 *}$, Ji-long Hao ${ }^{{ }^{\bowtie}}$, Cheng-wei Lu ${ }^{1 凶}$ \\ 1. Department of Ophthalmology, \\ 2. Department of Radiology, The First Hospital of Jilin University, No. 71 of xinmin St., Changchun, Jilin Province, 130021, China. \\ *Nan Guo, Xiu-fen Liu, Om Prakash Pant, Dan-Dan Zhou are co-first authors. \\ $\triangle$ Corresponding authors: Cheng-wei Lu, M.D., Ph.D., Department of Ophthalmology, the First Hospital of Jilin University, No. 71 of xinmin St., Changchun, \\ Jilin Province, 130021, China. Email address: lcwchina800@sina.com Telephone No: 8618684317115 and Ji-long Hao M.D., Ph.D., Department of Ophthalmology, \\ the First Hospital of Jilin University, No. 71 of xinmin St., Changchun, Jilin Province, 130021, China. Email address: 289736582@qq.com \\ (C) Ivyspring International Publisher. This is an open access article distributed under the terms of the Creative Commons Attribution (CC BY-NC) license \\ (https://creativecommons.org/licenses/by-nc/4.0/). See http://ivyspring.com/terms for full terms and conditions.
}

Received: 2018.09.06; Accepted: 2019.02.08; Published: 2019.03.10

\begin{abstract}
Circular RNAs (circRNAs) are a novel class of endogenous non-coding RNAs produced by back-splicing. They are found to be expressed in eukaryotic cells and play certain roles in various cellular functions, including fibrosis, cell proliferation, differentiation, apoptosis and angiogenesis. Dysregulated circRNAs are found in several human disorders including, malignancy, vascular, inflammatory as well as nervous diseases. Although, increasing evidence suggests that circRNAs may also contribute in different ocular diseases, the outline of circRNAs in ocular diseases remains obscure. In this review we consider the current state of knowledge regarding the potential role and underlying mechanism of circRNAs in ocular diseases including pterygium, age-related cataract, glaucoma, diabetic retinopathy, retinoblastoma, retinal vascular dysfunction and hyperhomocysteinemia induced ocular diseases, emphasizing that circRNAs could be promising biomarkers for the diagnosis and prognosis evaluation. Future circRNAs-targeted intervention may become a novel therapeutic tool for the treatment of ocular diseases.
\end{abstract}

Key words: circRNAs, non-coding RNAs, ocular diseases

\section{Introduction}

Long non-coding RNAs (lncRNAs) are described as RNAs with a minimum of 200 nucleotides in size and doesn't encode proteins. Currently, more than 28,000 long noncoding RNAs (lncRNAs) loci have been documented in the human genome [1]. These lncRNAs genes are usually situated in genomes either in an independent form or coinciding in compound forms alongside other genes [2]. Besides the linear expression found in most genes, messenger RNA (mRNA) and classical lncRNAs transcripts, cells also express circular RNAs (circRNAs) [3].

CircRNAs are a new group of endogenous non-coding RNAs with covalently sealed continuous loop structures and broadly expressed in eukaryotes, including mammals. They are produced either by exon skipping mechanism or by linking of the $5^{\prime}$ splice position with the $3^{\prime}$ splice position via back-splicing [4]. CircRNAs are abundantly expressed in a tissue-specific as well as developmental stage-specific design and play a significant part in various cellular functions, including fibrosis, cell proliferation, differentiation, apoptosis, and angiogenesis $[5,6]$. Moreover, these circular molecules can regulate gene expression by functioning as micro RNA (miRNA) sponges, RNA-binding protein repossessing mediators, or regulators of transcription [7, 8]. Abnormally expressed circRNAs were found in numerous human diseases including, malignancy, vascular, inflammatory and nervous diseases [9-11].

CircRNAs show a diverse expression outline in 
different eye diseases. The mechanism of circRNAs in the ocular diseases is simply emerging. Accumulative evidence had shown that abnormal expression of circRNAs is very closely related with the development, pathogenesis and progression of various ocular disorders [12-14]. Still, their clinical importance in ocular diseases remains concealed. In this review we will consider the current state of knowledge regarding the expression, regulation and the functional aspect of circRNAs in various ocular diseases (Table 1). In addition, circRNAs might be a potentially biomarker for diagnosis along with prognosis evaluation and proper intervention of circRNAs regulation could be a promising therapeutic target for various ocular diseases.

\section{Pterygium}

Pterygium is a benign, wing-shaped, common degenerative ocular surface disorder with a high incidence worldwide. The lesion can significantly interfere with vision when there is an encroachment of hyperplastic conjunctival tissue onto the cornea and sometimes become inflamed, leading to discomfort, redness and cosmetic difficulties [15]. It has been revealed that abnormal cell proliferation, defects in wound healing, angiogenesis and cell transformation were linked to the development and advancement of this disease [16]. However, the precise etiology and pathogenesis of pterygium are still unknown.

Table 1. Selected studies on the relationship between circRNAs and ocular diseases.

\begin{tabular}{|c|c|c|c|c|c|}
\hline Ocular diseases & CircRNA & Expression & $\begin{array}{l}\text { Related } \\
\text { molecules }\end{array}$ & Phenotypes affected & References \\
\hline Pterygium & $\begin{array}{l}\text { circ_0085020 } \\
\text { (circ-LAPTM4B) }\end{array}$ & $\begin{array}{l}\text { Up-regulated in pterygium } \\
\text { samplers. }\end{array}$ & Bcl-2 & $\begin{array}{l}\text { Affect viability, proliferation, migration, } \\
\text { apoptosis of pterygium and epithelial cells in } \\
\text { vitro. }\end{array}$ & [17] \\
\hline ARC & circHIPK3 & $\begin{array}{l}\text { Down-regulated in ARC lens } \\
\text { capsules and in all three } \\
\text { subtypes of ARC patients. }\end{array}$ & $\begin{array}{l}\text { a-SMA } \\
\text { miR-193a } \\
\text { CRYAA } \\
\text { E-cadherin ZO-1 }\end{array}$ & $\begin{array}{l}\text { Affect cell viability, proliferation and apoptosis } \\
\text { of primary cultured HLECs in vitro via } \\
\text { circHIPK3/miR-193a/CRYAA network. }\end{array}$ & {$[20]$} \\
\hline \multirow[t]{2}{*}{$\begin{array}{l}\text { Retinal } \\
\text { neurodegenerative } \\
\text { diseases }\end{array}$} & cZNF609 & $\begin{array}{l}\text { Up-regulated in rat retinas and } \\
\text { aqueous humor of } \\
\text { glaucomatous rat models' } \\
\text { eyes. }\end{array}$ & $\begin{array}{l}\text { miR-615, } \\
\text { METRN }\end{array}$ & $\begin{array}{l}\text { Affect retinal reactive gliosis, glial cell activation, } \\
\text { and RGCs survival in rat models of glaucoma in } \\
\text { vivo. Regulate Müller cell function directly and } \\
\text { RGCs function indirectly in vitro. Regulate } \\
\text { Müller cell through cZNF609/miR-615/METRN } \\
\text { network in vitro. }\end{array}$ & [26] \\
\hline & cZRANB1 & $\begin{array}{l}\text { Up-regulated in glaucomatous } \\
\text { rat models' retina and in the } \\
\text { aqueous humor from the } \\
\text { patients with POAG. }\end{array}$ & $\begin{array}{l}\text { miR-217, } \\
\text { RUNX2 }\end{array}$ & $\begin{array}{l}\text { Affect retinal reactive gliosis, glial cell activation } \\
\text { and RGC survival in rat models of glaucoma in } \\
\text { vivo. Regulate Müller cell function directly and } \\
\text { RGCs function indirectly in vitro. Regulate } \\
\text { Müller cell through cZNF609/miR-615/METRN } \\
\text { network in vitro. }\end{array}$ & [27] \\
\hline \multirow[t]{2}{*}{ DR } & circHIPK3 & $\begin{array}{l}\text { Up-regulated in diabetic retina } \\
\text { and retinal endothelial cells } \\
\text { following stressors related to } \\
\text { diabetes mellitus. }\end{array}$ & $\begin{array}{l}\text { miR-30a-3p } \\
\text { VEGFC, FZD4, } \\
\text { WNT2 }\end{array}$ & $\begin{array}{l}\text { Affect retinal endothelial cell viability, } \\
\text { proliferation, migration, and tube formation in } \\
\text { HRVECs in vitro via circHIPK3-miR-30a-3p- } \\
\text { VEGFC/ WNT2/FZD4 network. Affect retinal } \\
\text { vascular dysfunction, vascular leakage, and } \\
\text { inflammation in Diabetes Mellitus-induced mice } \\
\text { models in vivo via circHIPK3-miR-30a-3p- } \\
\text { VEGFC/WNT2/FZD4 network. }\end{array}$ & [13] [47] \\
\hline & Circ_0005015 & $\begin{array}{l}\text { Up-regulated in the plasma, } \\
\text { vitreous sample, and } \\
\text { fibrovascular membranes of } \\
\text { DR patients. }\end{array}$ & $\begin{array}{l}\text { miR-519d-3p, } \\
\text { MMP-2, XIAP, } \\
\text { STAT3 }\end{array}$ & $\begin{array}{l}\text { Regulate endothelial cell proliferation, migration } \\
\text { and tube formation of HRVECs in vitro via } \\
\text { circ-0005015-miR519d-3p-MMP-2/STAT3/XIAP } \\
\text { network. }\end{array}$ & {$[30]$} \\
\hline RB & hsa_circ_0001649 & $\begin{array}{l}\text { Down-regulated in RB } \\
\text { samples and cells. }\end{array}$ & $\begin{array}{l}\text { Bcl-2,caspase-3 } \\
\text { p-AKT, p-mTOR }\end{array}$ & $\begin{array}{l}\text { Affect cell growth and apoptosis in RB cells in } \\
\text { vitro via AKT/mTOR signaling pathway. Affect } \\
\text { xenograft growth in vivo. }\end{array}$ & {$[34]$} \\
\hline $\begin{array}{l}\text { Vascular } \\
\text { endothelial } \\
\text { dysfunction }\end{array}$ & cZNF609 & $\begin{array}{l}\text { Up-regulated in retinas of } \\
\text { diabetic mice and OIR mice at } \\
\text { the neovascularization stage. }\end{array}$ & $\begin{array}{l}\text { miR-615-5p, } \\
\text { MEF2A }\end{array}$ & $\begin{array}{l}\text { Affect endothelial cell viability, migration, tube } \\
\text { formation and apoptosis in diabetic and OIR } \\
\text { mice models in vivo. Regulate capillary } \\
\text { degeneration and vascular leakage and retinal } \\
\text { inflammation response of endothelial cells in } \\
\text { vitro via cZNF609/miR-615-5p/MEF2A network. }\end{array}$ & [38] \\
\hline $\begin{array}{l}\text { Hyperhomocysthei } \\
\text { nemia induced } \\
\text { ocular diseases }\end{array}$ & $\begin{array}{l}\text { mmu_circRNA_21649 } \\
\text { mmu_circRNA_33761 } \\
\text { mmu_circRNA_008614 } \\
\text { mmu_circRNA_29109 }\end{array}$ & $\begin{array}{l}\text { Down-regulated in the eyes of } \\
\text { CBS lacking mice models. } \\
\text { Up-regulated in the eyes of } \\
\text { CBS lacking mice models. }\end{array}$ & $\begin{array}{l}\text { Grm1 } \\
\text { Bbs5 } \\
\text { Mlip } \\
\text { Sp1 }\end{array}$ & $\begin{array}{l}\text { Mitrocondrial dysfunction, inflammation, redox } \\
\text { imbalance }\end{array}$ & {$[44]$} \\
\hline
\end{tabular}

Abbreviations: ARC, Age-related cataract; DR, Diabetic retinopathy; RB, Retinoblastoma; Bcl-2, B-cell lymphoma-2; a-SMA, a-smooth muscle actin; ZO-1, Zonula occludens-1; RUNX2, Runt-related transcription factor 2; FZD4, Frizzled 4 gene; MMP2, matrix metallopeptidase 2; XIAP, X-linked inhibitor of apoptosis protein; STAT3, signal transducer and activator of transcription 3; MEF2A, Myocyte-specific enhancer factor 2A. CRYAA: a gene that encodes aA lens protein; METRN: Meteorin, glial cell differentiation Regulator; WNT2: Wnt family member 2, the WNT gene family consists of structurally related genes which encode secreted signaling proteins; MMP-2: matrix metallopeptidase 2, a member of the matrix metalloproteinase (MMP) gene family; RGC: retinal ganglion cell; POAG: primary open-angle glaucoma; VEGFC: vascular endothelial growth factor-C; OIR: oxygen-induced retinopathy: HRVECs: human retinal vascular endothelial cells; HRVECs: human retinal vascular endothelial cells. 
$\mathrm{Li}$ et al. suggested that aberrant expression of nearly 669 kinds of circRNAs were discovered in pterygium [17]. Among them, circ_0085020 (circLAPTM4B) was considerably up-regulated in pterygium samples, and it was demonstrated by circ_0085020 silencing that circ_0085020 promoted the proliferation as well as the migration of pterygium fibroblasts, and inhibited UV-induced apoptosis of pterygium epithelial cells suggesting that this circRNAs could be a promising biomarker for the management of pterygium [17].

\section{Age-related cataract}

Age-related cataract (ARC) is the most cause of vision loss among the aged population [18]. Surgery is the only effective treatment option for ARC [19]. However, the high surgical cost has brought huge financial burden to the society. Accumulating studies had clarified that the circRNAs play a vital role in ocular diseases, while the functions of circRNAs in $A R C$ remains to be discovered.

Liu et al. revealed that down-regulation of circHIPK3 expression was found in all three subtypes of ARC compared with the control [20]. In vitro study had discovered that the functions of the human lens epithelial cells (HLECs) were regulated by the circHIPK3/miR-193a/CRYAA network. The downregulation of circHIPK3 could lead to the overexpression of miR-193a, then operating on CRYAA. CRYAA is a completely novel target gene of miR-193a in HLECs and closely associated with the preservation of lens clarity. While the balance of a-cyrstallins expression was broken-down via this network, the protective effect of a-cyrstallins would be destroyed. Additionally, circHIPK3 silencing in ARC cases stimulated the HLECs apoptosis mediated by oxidative stress [20].

In conclusion, the role of circHIPK3 as a functional regulator of HLECs via circHIPK3/miR193a/CRYAA showed a new targeted method for the prevention as well as treatment for ARC.

\section{Retinal neurodegenerative diseases}

Glaucoma is a progressive retinal neurodegenerative disease characterized by the degeneration of retinal ganglion cells. It is a major cause of irreversible visual impairment worldwide. The intraocular pressure (IOP) level plays a vital role in the degeneration of retinal ganglion cells [21]. In recent years, circRNAs have come into sight as possible regulators in some neurodegenerative disorders $[13,22,23]$. Though, the exact molecular mechanism of circRNAs in glaucoma induced retinal neurodegenerative progression is still unclear [24, 25].

Wang et al. suggested that cZNF609 expression was significantly increased in the glaucoma induced retinal neurodegeneration in rat models [26]. CZNF609 silencing eventually protected retinal ganglion cells from the damage triggered by high IOP level and restrained retinal reactive gliosis by directly regulating Müller cell function. CZNF609 was also found to act as a miR-615 sponge and hinder miR-615 activity, resulting in increased METRN, which partly reversed cZNF609 silencing-mediated inhibitory effects on the cell proliferation of retinal glial cells [26].

In another study, cZRANB1 expression was also found to be dramatically upregulated in retinal neurodegeneration in glaucoma rat model induced by chamber injection of microbeads [27]. CZRANB1 knockdown by short hairpin RNA (shRNAs) hindered retinal glial cell activation, gliosis, and restored RGC survival in vivo. Additionally, cZRANB1 knockdown indirectly regulated retinal ganglion cells function by directly regulating the Müller cells' function in vitro. cZRANB1 was proved to act as a miRNA sponge, and regulate Müller cells' function via cZRANB1/miR-217/RUNX2 signaling [27]. Furthermore, Han et al. detected circRNAs in the postnatal rat retina respectively at day P3, P7, and P12. The changes of these circRNAs species were analyzed and some of them were associated with neuronal apoptosis in the developing nervous system [12].

These results suggested that circRNAs (cZNF 609 and CZRANB1) play an important role in retinal neurodegeneration. CZNF609/miR-615/METRN and CZRANB1-oriented treatments may serve as a potential therapeutic target for the treatment of retinal neurodegenerative diseases.

\section{Diabetic Retinopathy}

Diabetic retinopathy (DR) is the major microvascular complication of uncontrolled diabetes mellitus and also the leading cause of visual impairment and blindness worldwide [28]. Existing worldwide prevalence of DR is almost $30 \%$ of the patient with diabetes and around $50 \%$ people have sight-threatening DR [29]. Despite various treatment options, most cases with DR don't respond well to existing therapeutic methods. Hence, it is necessary to focus on specific biomarkers for the diagnosis and treatment of diabetic retinopathy.

Circ_0005015 was markedly overexpressed in the plasma fraction, vitreous samples and preretinal fibrovascular membranes (FVMs) of diabetic retinopathy (DR) patients [30]. Circ_0005015 silencing evidently inhibited the spheroid sprouting, migration as well as tube formation of HRVECs [30]. Moreover, circ_0005015 might regulated the function of HRVEC 
as a sponge of miR-519d-3p in vitro, which was closely associated with cell growth, migration and proliferation via acting on the target matrix metalloproteinase (MMP)-2, STAT3, or XIAP genes [30]. MiR-519d-3p mimic transfection was discovered to decrease the expression level of MMP-2, STAT3, along with XIAP in HRVECs, and further reduced proliferation, migration and tube formation of HRVECs [30]. Further, it was explored that 30 circRNAs were dramatically overexpressed in the serum samples of DR patients compared with the serum samples from the control group [14]. However, in vivo and in vitro studies are required to explain the mechanism of circRNA-mediated DR development. In conclusion, circRNAs participates in the pathogenesis of DR and thus aid as a potential biomarker for the diagnosis and molecular targets for the treatment of DR.

\section{Retinoblastoma}

Retinoblastoma (RB), a malignant intraocular tumor originating from embryonic retinal cells, is a sight and life-threatening disease in children, especially infants. Tumor formation is initiated from the alleles mutation of the retinoblastoma tumor suppressor gene RB1 which is located at 13q14 [31]. $\mathrm{AKT}$, a kind of protein kinase, and its downstream effector mTOR were found in numerous regulated signaling pathways related to cancers [32]. Even though AKT/mTOR was discovered in RB progression, the relationship between AKT and circRNAs is still unclear [33].

Recently, hsa_circ_0001649 was found evidently downregulated in a sample from tumor tissues as well as in most RB cell lines [34]. The expression level of hsa_circ_0001649 was closely linked with the tumor size, clinical stages, pathological type and overall survival [34]. Hsa_circ_0001649 was enhanced in Y79 cells and knocked down in WERI-Rb1 cells to verify the biological roles of hsa_circ_0001649 in RB [34]. The results showed that the low expression level of hsa_circ_0001649 acted as a promotor in the progression of RB by regulating cell growth and cell apoptosis in vitro. And in vivo studies, the transplanted tumor in up-regulated hsa_circ_0001649 group grew obviously slower and the tumors separated from the nude mice weighed much less as compared to the blank vector group [34]. Still, further target genes regulated by hsa_circ_0001649 are required for further investigation.

Furthermore, hsa_circ_0001649 was found to regulate the cell apoptosis and proliferation by AKT/ mTOR signaling pathway [34]. To sum up, h sa_circ_ 0001649 might be a possibly prognostic biomarker as well as the treatment target for RB.

\section{Retinal vascular dysfunction}

Ischemic retinopathies, including retinopathy of prematurity (ROP) and diabetic retinopathy (DR), share many similar pathological characteristics such as blood vessel injury and consequential pathological angiogenesis. Vascular dysfunction is commonly related to endothelial cell dysfunction and abnormal gene regulation $[35,36]$. Hence, it is essential to reveal the exact biomarker for retinal vascular dysfunction for the prevention and management of vascular complications. It was reported that circRNAs of SIAE and ZNF280C along with other molecules and pathway plays an important part in the progression of ROP [37]. However, the precise mechanism is obscured.

Liu et al. suggested that cZNF609 was dramatically overexpressed under the high glucose condition and oxygen-induced retinopathy (OIR) both in vivo and in vitro [38]. CZNF609 silencing considerably reduced capillary degeneration, release of inflammatory factors, decreased retinal vessel loss and suppressed pathological angiogenesis in vivo. Alternatively, cZNF609 silencing increased the cell viability, apoptosis, proliferation, migration as well as tube formation, and protected the endothelial cell against oxidative as well as hypoxia stress in vitro [38]. Moreover, cZNF609 operated as an endogenous miR$615-5 p$ sponge to sequester inhibiting miR-615-5p action which leads to MEF2A overexpression. MEF2A overexpression could release cZNF609 silencingmediated outcomes on endothelial cell migration, tube formation, as well as apoptosis [38]. These results suggested that the network consisting of cZNF609, miR-615-5p and MEF2A were connected with the retinal vascular dysfunction.

\section{Hyperhomocysteinemia induced ocular diseases}

Hyperhomocysteinemia (HHcy) is a metabolic disorder characterized by increased level of homocysteine in plasma due to the deficiency of Cystathionine- $\beta$-synthase (CBS) and less commonly due to deficiencies of enzymes involved in de novo methionine synthesis [39, 40]. Regardless of various vascular diseases including coronary, cerebral and peripheral vascular dysfunction, HHcy can result in various ocular diseases such as retinovascular thromboembolic disease and ectopia lentis along with vascular cognitive impairment [41-43]. However, exact mechanism involved in the development of the ocular diseases is obscured.

Singh et al. revealed that 74 circRNAs showed distinction expression profile, out of which nearly $27 \%$ were down-regulated while almost $73 \%$ were 
up-regulated in the eyes of CBS lacking mice models [44]. They also revealed that HHcy could possibly disrupt the complete metabolism of a cell by altering the methylation of key target genes' regulatory elements and influenced the level of gene products as well as disease phenotype by modulating the "genesmRNAs-miRNAs-circRNAs-proteins" axis. Furthermore, miRNAs regulated gene expressions by inhibiting mRNA translation and circRNAs and miRNAs interact with each other to regulate miRNA functions suggesting that circRNAs might also be involved in various aspects of the ocular biology [44]. In conclusion, circRNAs might play a role in the development and progression of various eye disorders related with homocysteinemia and could be a potential diagnostic as well as the therapeutic target in homocysteinemia induced ocular diseases.

\section{Conclusion}

For decades, circRNAs were mistakenly considered as the transcriptional oddities of only limited biological importance[45] [46]. With the development of technologies, circRNAs becomes an innovative issue, more and more researches related to the significance of circRNAs with human disease have been stated. Increasing evidence suggests that circRNAs, being a novel class of endogenous non-coding RNAs, may play an important role in the pathogenesis as well as disease progression in various ocular disease including pterygium, age-related cataract, glaucoma, diabetic retinopathy, retinoblastoma, retinal vascular dysfunction and homocysteinemia induced ocular diseases. CircRNAs had shown different roles and expression patterns in different eye diseases. Dysregulation of circRNAs has been seemed to link with a wide range of biological processes, such as viability, tube formation, apoptosis, and proliferation. However, aberrantly expressed circRNAs may be driven by the same promoters of linear RNAs, possibility of off target effects in siRNA. And the techniques used to identify and validate circRNAs are largely experimental and approaches remain to be standardized. Further in-depth studies are needed. To sum up, circRNAs may aid as a potential biomarker for diagnosis and a prognostic evaluator in ocular diseases. Additionally, circRNAs-target interventions might be a promising therapy against related ocular diseases.

\section{Competing Interests}

The authors have declared that no competing interest exists.

\section{References}

1. Fong ST, Stanisich VA. Location and characterization of two functions on RP1 that inhibit the fertility of the IncW plasmid R388. J Gen Microbiol. 1989; 135: 499-502.

2. Ulitsky I. Evolution to the rescue: using comparative genomics to understand long non-coding RNAs. Nat Rev Genet. 2016; 17: 601-14.

3. Holdt LM, Kohlmaier A, Teupser D. Molecular functions and specific roles of circRNAs in the cardiovascular system. Noncoding RNA Res. 2018; 3: 75-98.

4. Jeck WR, Sharpless NE. Detecting and characterizing circular RNAs. Nat Biotechnol. 2014; 32: 453-61.

5. Vicens Q, Westhof E. Biogenesis of Circular RNAs. Cell. 2014; 159: 13-4.

6. Liu J, Liu T, Wang X, He A. Circles reshaping the RNA world: from waste to treasure. Mol Cancer. 2017; 16: 58

7. Chen LL. The biogenesis and emerging roles of circular RNAs. Nat Rev Mol Cell Biol. 2016; 17: 205-11

8. Salzman J. Circular RNA Expression: Its Potential Regulation and Function. Trends Genet. 2016; 32: 309-16.

9. Li H, Hao X, Wang H, Liu Z, He Y, Pu M, et al. Circular RNA Expression Profile of Pancreatic Ductal Adenocarcinoma Revealed by Microarray. Cell Physiol Biochem. 2016; 40: 1334-44.

10. Lukiw WJ. Circular RNA (circRNA) in Alzheimer's disease (AD). Front Genet. 2013; 4: 307.

11. Fu L, Yao T, Chen Q, Mo X, Hu Y, Guo J. Screening differential circular RNA expression profiles reveals hsa_circ_0004018 is associated with hepatocellular carcinoma. Oncotarget. 2017; 8: 58405-16.

12. Han J, Gao L, Dong J, Bai J, Zhang M, Zheng J. The expression profile of developmental stage-dependent circular RNA in the immature rat retina. Molecular vision. 2017; 23: 457-69.

13. Shan K, Liu C, Liu BH, Chen X, Dong R, Liu X, et al. Circular Noncoding RNA HIPK3 Mediates Retinal Vascular Dysfunction in Diabetes Mellitus. Circulation. 2017; 136: 1629-42.

14. Gu Y, Ke G, Wang L, Zhou E, Zhu K, Wei Y. Altered Expression Profile of Circular RNAs in the Serum of Patients with Diabetic Retinopathy Revealed by Microarray. Ophthalmic Res. 2017; 58: 176-84.

15. Chui J, Di Girolamo N, Wakefield D, Coroneo MT. The pathogenesis of pterygium: current concepts and their therapeutic implications. Ocul Surf. 2008; 6: 24-43.

16. Liu T, Liu Y, Xie L, He X, Bai J. Progress in the pathogenesis of pterygium. Curr Eye Res. 2013; 38: 1191-7.

17. Li XM, Ge HM, Yao J, Zhou YF, Yao MD, Liu C, et al. Genome-Wide Identification of Circular RNAs as a Novel Class of Putative Biomarkers for an Ocular Surface Disease. Cell Physiol Biochem. 2018; 47: 1630-42.

18. Abdelkader H, Alany RG, Pierscionek B. Age-related cataract and drug therapy: opportunities and challenges for topical antioxidant delivery to the lens. J Pharm Pharmacol. 2015; 67: 537-50.

19. Song E, Sun $\mathrm{H}, \mathrm{Xu} \mathrm{Y,} \mathrm{Ma} \mathrm{Y,} \mathrm{Zhu} \mathrm{H,} \mathrm{Pan} \mathrm{CW.} \mathrm{Age-related} \mathrm{cataract,} \mathrm{cataract}$ surgery and subsequent mortality: a systematic review and meta-analysis. PLoS One. 2014; 9: e112054.

20. Liu X, Liu B, Zhou M, Fan F, Yu M, Gao C, et al. Circular RNA HIPK3 regulates human lens epithelial cells proliferation and apoptosis by targeting the miR-193a/CRYAA axis. Biochem Biophys Res Commun. 2018.

21. Weinreb RN, Aung T, Medeiros FA. The pathophysiology and treatment of glaucoma: a review. JAMA. 2014; 311: 1901-11.

22. Guo JU, Agarwal V, Guo H, Bartel DP. Expanded identification and characterization of mammalian circular RNAs. Genome Biol. 2014; 15: 409.

23. Lasda E, Parker R. Circular RNAs: diversity of form and function. RNA. 2014; 20: $1829-42$

24. Lu D, Xu AD. Mini Review: Circular RNAs as Potential Clinical Biomarkers for Disorders in the Central Nervous System. Front Genet. 2016; 7: 53.

25. Kumar L, Shamsuzzama, Haque R, Baghel T, Nazir A. Circular RNAs: the Emerging Class of Non-coding RNAs and Their Potential Role in Human Neurodegenerative Diseases. Mol Neurobiol. 2017; 54: 7224-34.

26. Wang JJ, Liu C, Shan K, Liu BH, Li XM, Zhang SJ, et al. Circular RNA-ZNF609 regulates retinal neurodegeneration by acting as miR-615 sponge. Theranostics. 2018; 8: 3408-15.

27. Wang JJ, Shan K, Liu BH, Liu C, Zhou RM, Li XM, et al. Targeting circular RNA-ZRANB1 for therapeutic intervention in retinal neurodegeneration. Cell Death Dis. 2018; 9: 540

28. Yau JW, Rogers SL, Kawasaki R, Lamoureux EL, Kowalski JW, Bek T, et al. Global prevalence and major risk factors of diabetic retinopathy. Diabetes Care. 2012; 35: 556-64

29. Zheng Y, He M, Congdon N. The worldwide epidemic of diabetic retinopathy. Indian J Ophthalmol. 2012; 60: 428-31.

30. Zhang SJ, Chen X, Li CP, Li XM, Liu C, Liu BH, et al. Identification and Characterization of Circular RNAs as a New Class of Putative Biomarkers in Diabetes Retinopathy. Invest Ophthalmol Vis Sci. 2017; 58: 6500-9.

31. Mendoza PR, Grossniklaus HE. The Biology of Retinoblastoma. Prog Mol Biol Transl Sci. 2015; 134: 503-16.

32. Qin G, Li P, Xue Z. Triptolide induces protective autophagy and apoptosis in human cervical cancer cells by downregulating Akt/mTOR activation. Oncol Lett. 2018; 16: 3929-34.

33. Chakraborty S, Khare S, Dorairaj SK, Prabhakaran VC, Prakash DR, Kumar A. Identification of genes associated with tumorigenesis of retinoblastoma by microarray analysis. Genomics. 2007; 90: 344-53. 
34. Xing L, Zhang L, Feng Y, Cui Z, Ding L. Downregulation of circular RNA hsa_circ_0001649 indicates poor prognosis for retinoblastoma and regulates cell proliferation and apoptosis via AKT/mTOR signaling pathway. Biomed Pharmacother. 2018; 105: 326-33.

35. Michalik KM, You X, Manavski Y, Doddaballapur A, Zornig M, Braun T, et al. Long noncoding RNA MALAT1 regulates endothelial cell function and vessel growth. Circ Res. 2014; 114: 1389-97.

36. Yan B, Yao J, Liu JY, Li XM, Wang XQ, Li YJ, et al. IncRNA-MIAT regulates microvascular dysfunction by functioning as a competing endogenous RNA. Circ Res. 2015; 116: 1143-56.

37. Yang Y, Pan JJ, Zhou XG, Zhou XY, Cheng R. Differentially expressed miRNAs in premature infants with retinopathy-a bioinformatics analysis. Int J Ophthalmol. 2018; 11: 773-9.

38. Liu C, Yao MD, Li CP, Shan K, Yang H, Wang JJ, et al. Silencing Of Circular RNA-ZNF609 Ameliorates Vascular Endothelial Dysfunction. Theranostics. 2017; 7: 2863-77.

39. Ueland PM, Refsum H. Plasma homocysteine, a risk factor for vascular disease: plasma levels in health, disease, and drug therapy. J Lab Clin Med. 1989; 114: 473-501.

40. Selhub J, Miller JW. The pathogenesis of homocysteinemia: interruption of the coordinate regulation by S-adenosylmethionine of the remethylation and transsulfuration of homocysteine. Am J Clin Nutr. 1992; 55: 131-8.

41. Reis RP, Luis AS. [Homocysteinemia and vascular disease--a new risk factor is born]. Rev Port Cardiol. 1999; 18: 507-14.

42. Couser NL, McClure J, Evans MW, Haines NR, Burden SK, Muenzer J. Homocysteinemia due to MTHFR deficiency in a young adult presenting with bilateral lens subluxations. Ophthalmic Genet. 2017; 38: 91-4.

43. Dong N, Wang B, Chu L, Xiao L. Plasma homocysteine concentrations in the acute phase after central retinal vein occlusion in a Chinese population. Curr Eye Res. 2013; 38: 1153-8.

44. Singh M, George AK, Homme RP, Majumder A, Laha A, Sandhu HS, et al. Circular RNAs profiling in the cystathionine-beta-synthase mutant mouse reveals novel gene targets for hyperhomocysteinemia induced ocular disorders. Exp Eye Res. 2018; 174: 80-92.

45. Nigro JM, Cho KR, Fearon ER, Kern SE, Ruppert JM, Oliner JD, et al. Scrambled exons. Cell. 1991; 64: 607-13.

46. Cocquerelle C, Mascrez B, Hetuin D, Bailleul B. Mis-splicing yields circular RNA molecules. FASEB J. 1993; 7: 155-60.

47. Andrea C, Maria PG. Update on the regulation of HIPK1, HIPK2 and HIPK3 protein kinases by microRNAs. MicroRNA (Shariqah, United Arab Emirates). 2018. 